\title{
Effectiveness of Rotigotine plus intensive and goal-based rehabilitation versus Rotigotine alone in "de-novo" Parkinsonian subjects: a randomized controlled trial with 18-month follow-up
}

\author{
D. Ferrazzoli ${ }^{1}$ P. Ortelli ${ }^{1} \cdot$ G. Riboldazzi ${ }^{2,3} \cdot$ R. Maestri ${ }^{4} \cdot$ G. Frazzitta $^{1}$
}

Received: 29 December 2017 / Revised: 7 February 2018 / Accepted: 8 February 2018 / Published online: 13 February 2018

(c) The Author(s) 2018. This article is an open access publication

\begin{abstract}
Background Dopamine Replacement Therapy (DRT) represents the most effective treatment for Parkinson's disease (PD). Nevertheless, several symptoms are unresponsive to treatment and its long-term use leads to serious side effects. To optimize the pharmacological management of PD, dopamine-agonists are often prescribed to "de-novo" patients. Moreover, several studies have shown the effectiveness and the synergic effect of rehabilitation in treating PD.

Objective To evaluate the synergism between DRT and rehabilitation in treating PD, by investigating the short and the longterm effectiveness of a multidisciplinary, intensive and goal-based rehabilitation treatment (MIRT) in a group of patients treated with Rotigotine.

Materials and methods In this multicenter, single blinded, parallel-group, 1:1 allocation ratio, randomized, non-inferiority trial, 36 "de-novo" PD patients were evaluated along 18 months: 17 were treated with Rotigotine plus MIRT; 19 were treated with Rotigotine alone (R). The primary outcome measure was the total score of Unified Parkinson's Disease Rating Scale (UPDRS). The secondary outcomes included the UPDRS sub-sections II and III (UPDRS II-III), the 6-Minute Walk Test (6MWT), the Timed Up and Go Test (TUG) and the amount of Rotigotine. Patients were evaluated at baseline (T0), 6 months (T1), 1 year (T2), and at 18 months (T3).

Results No differences in UPDRS scores in the two groups (total score, III part and II part, $p=0.48, p=0.90$ and $p=0.40$, respectively) were found in the time course. Conversely, a greater improvement in Rotigotine + MIRT group was observed for 6MWT $(p<0.0001)$ and TUG $(p=0.03)$. Along time, the dosage of Rotigotine was higher in patients who did not undergo MIRT, at all observation times following T0.

Conclusions Over the course of 18 months, the effectiveness of the combined treatment (Rotigotine + MIRT) on the patients' global clinical status, evaluated with total UPDRS, was not inferior to that of the pharmacological treatment with Rotigotine alone. Importantly, rehabilitation allowed patients to gain better motor performances with lower DRT dosage.
\end{abstract}

Keywords Parkinson's disease $\cdot$ Rehabilitation $\cdot$ Multidisciplinary care $\cdot$ Dopamine agonists $\cdot$ DRT-related side effects

D. Ferrazzoli and P. Ortelli have contributed equally to this work.

D. Ferrazzoli

davideferrazzoli@gmail.com

1 Department of Parkinson's Disease, Movement Disorders and Brain Injury Rehabilitation, "Moriggia-Pelascini”" Hospital-Via Pelascini 3, Gravedona ed Uniti (CO), 22015 Como, Italy

2 Parkinson's Disease and Movement Disorders Center, ASST dei Sette Laghi, Varese, Italy
3 Parkinson's disease Rehabilitation Center, Fondazione Borghi, Brebbia, Varese, Italy

4 Department of Biomedical Engineering, Istituti Clinici Scientifici Maugeri Spa Società Benefit, IRCCS, Via per Montescano 3, Montescano, 27040 Pavia, Italy 


\section{Introduction}

Parkinson's disease (PD) is one of the most common progressive neurodegenerative diseases. It is classically defined as a disorder related to degeneration of dopamine producing neurons in the pars compacta of the substantia nigra. PD is typically characterized by dopamine-related motor disturbances such as tremor, rigidity and bradykinesia. The cornerstone of symptomatic treatment for PD is the dopamine replacement therapy (DRT), which works by restoring the physiological synaptic plasticity in the dopamine-denervated striatum [1]. L-dopa still remains the most effective drug for PD [2], but other dopaminergic agents, such as dopamine agonists (DAs), monoamine oxidase (MAO)-B inhibitors and catechol- $O$-methyl transferase (COMT) inhibitors are used in the clinical practice.

A relevant issue regarding DRT concerns its long-term use, as it might cause aberrant structural changes in the striatum leading to the development of motor and behavioural side effects (such as motor fluctuations, wearing-off, dyskinesias, dopamine dysregulation syndrome) [3], which worsen the patients' clinical conditions and the quality of their lives [4]. The total amount of DRT represents another aspect worthy of consideration: Warren Olanow et al. [5] described a relative 1-dopa threshold effect, and they found that the risk of developing dyskinesias and wearing-off was increased at l-dopa doses $\geq 400 \mathrm{mg} /$ day.

In order to optimize the pharmacological management of PD over time, the DAs are often prescribed to "denovo" patients [6]. Among all DAs, Rotigotine is the most recent and offers several potential advantages, including a rapid onset of action, a constant drug delivery and ease of use $[7,8]$.

While in early PD the predominant motor symptoms are dopamine-related, in the more advanced disease stages the involvement of other neurotransmitter systems [9] leads to the development of no dopamine-related disturbances, which are unresponsive to DRT [9].

Recently, several studies have shown the effectiveness of rehabilitation for PD [10-16], mainly on drug-resistant axial disturbances (such as postural and gait dysfunctions) [17-19]. Specific physical trainings may influence positively the neuroplasticity in the basal ganglia by modulating the cortico-striatal excitability, even in early PD stages [20-23]. In a previous study [14] we have demonstrated that a tailored, intensive, multidisciplinary and goal-based rehabilitation treatment (MIRT) slowed down the progression of motor symptoms and reduced the need for increasing DRT in a group of PD patients in early stage of disease.

Such kind of evidences confirms the relevance and the beneficial role of rehabilitation even in early PD.
We argued that the good synergism between an optimal DRT use and the rehabilitation efforts could be crucial for maximizing their positive effects on cortico-striatal plasticity and for reducing the occurrence of drug-related motor and behavioural side effects $[14,24]$.

In order to investigate and better define the potential advantages coming from the synergism between DRT and rehabilitation in treating $\mathrm{PD}$, in this study we have investigated the short- and the long-term (18 months) effectiveness of MIRT in a group of "de-novo" patients treated with rotigotine as monotherapy, in comparison with a control group of patients treated with the same DA, but who did not undergo rehabilitation.

\section{Materials and methods}

This was a multicenter, single blinded, parallel-group, 1:1 allocation ratio, randomized trial, aiming to compare the effectiveness of a treatment combining the use of the dopaminergic drug Rotigotine plus rehabilitation (Rotigotine + MIRT group) versus a pharmacological treatment alone, with the same DA (Rotigotine group) (see Fig. 1).

The hypothesis of this trial was that the synergistic association of the monotherapy with Rotigotine + MIRT, in a group of "de-novo" PD subjects, was, at least, as clinically effective as the Rotigotine alone, allowing at the same time a reduction in the need to increase the dopaminergic drug dosage along an 18-month observation period.

Hence, noninferiority of Rotigotine + MIRT with respect to Rotigotine alone was of interest on the premise that the first strategy might offer important advantages reducing the occurrence of drug-related motor and behavioural side effects. The study was, therefore, designed as a non-inferiority trial.

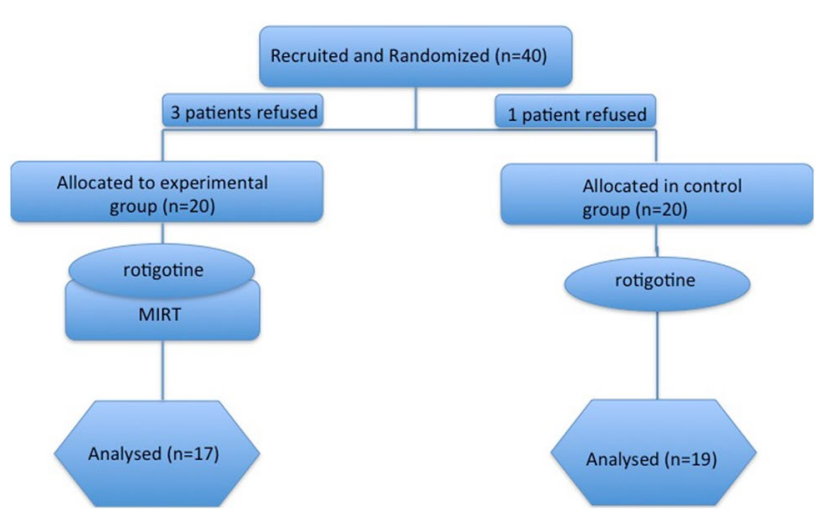

Fig. 1 Experimental design 


\section{Participants}

The present study was conducted at the Department of Parkinson's Disease, Movement Disorders and Brain Injury Rehabilitation of the "Moriggia-Pelascini" Hospital (Gravedona ed Uniti, Italy) and at the Parkinson's disease Rehabilitation Center, "Fondazione Borghi" (Brebbia, Italy).

Forty "de-novo" patients with a diagnosis of idiopathic PD were considered eligible from expert neurologists in movement disorders. 36 of these accepted to participate and concluded the study (17 in the experimental group and 19 in the control group). Eligibility criteria were the following: (i) diagnosis of idiopathic PD according to the UK Brain Bank criteria [25], (ii) Hoehn and Yahr stage 1-2. Exclusion criteria were: (i) rotigotine intolerance and/or need to change the rotigotine monotherapy with other dopaminergic medications during the study period, (ii) any focal brain lesion detected with brain imaging studies (CT or MRI), (iii) psychosis (evaluated with Neuropsychiatric Inventory), (iv) auditory, visual and/or vestibular dysfunctions, (v) presence of comorbidities impairing the autonomy in activities of daily living.

The sample size was calculated assuming the same change in total UPDRS between the enrollment and the end of the study for both approaches, with a non-inferiority margin equal to 4.5 (i.e. the minimal clinically important difference [26] to achieve a power of $80 \%$, with a onesided type 1 error rate of 5\%). This resulted in 32 patients (16 patients per group). Taking into account possible dropouts, a sample size of 40 was deemed to be appropriate for this trial. Enrolled patients were randomly assigned to the Rotigotine group or to the Rotigotine + MIRT group using a computer-generated list of random numbers: 20 patients to the Rotigotine condition (Control Group) and
20 patients to the Rotigotine + MIRT condition (Experimental Group). The sequence was concealed until assignment, and the people enrolling participants did not know in advance which treatment the patient was assigned.

All eligible patients began treatment with Rotigotine, at the same dosage of $2 \mathrm{mg} / 24 \mathrm{~h}$, which was then increased after 7 days to $4 \mathrm{mg} / 24 \mathrm{~h}$. 4 weeks from the onset of Rotigotine treatment, following the randomization list, the control group continued to be treated only with the pharmacological treatment, while the other (Rotigotine + MIRT) underwent a 4-week MIRT. The same MIRT was repeated 1 year later.

MIRT was performed at the Department of Parkinson's Disease, Movement Disorders and Brain Injury Rehabilitation of the "Moriggia-Pelascini" Hospital (Gravedona ed Uniti, Italy). Patients enrolled in the control group did not undergo any rehabilitation treatment during the same study period (see Fig. 2). The study design and protocol were approved by the by the local institutional review board and by the Central Ethics Committee and were in accordance with the code of Ethics of the World Medical Association (Declaration of Helsinki, 1967). All patients signed an informed written consent prior to the participation to the study for the use of their clinical data for scientific purposes. This trial was registered on ClinicalTrials.gov website (NCT02100176).

\section{Outcome measures}

Neurologists and physiotherapists expert in movement disorders, blinded to the treatment allocation and to the study design, assessed clinical, functional and motor scales. The primary outcome measure was the total score of Unified Parkinson's Disease Rating Scale (UPDRS). The secondary

Fig. 2 Study design

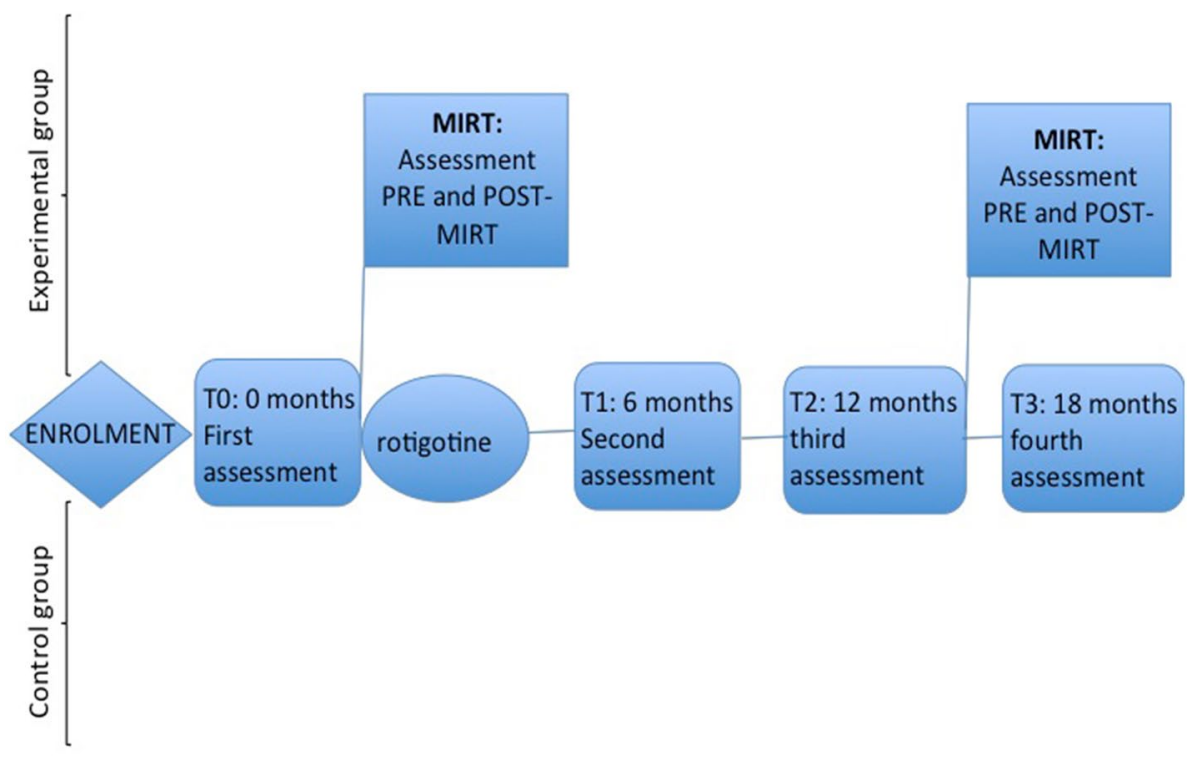


outcomes included the UPDRS sub-sections II and III (UPDRS II-III), the Six-Minute Walk Test (6MWT), the Timed Up and Go Test (TUG) and the amount of DRT. All patients were evaluated at baseline (T0), 6 months (T1), 1 year (T2), and at 18 months (T3) (see Fig. 2). All evaluations were performed, in the morning, at about 9 AM, $1 \mathrm{~h}$ after the rotigotine transdermal patch placement. The assessment took part in the centre that previously enrolled the patient. During the 18-month study, the neurologist modified the pharmacological dosage on the basis of the patients' clinical status and need. The dosage of rotigotine was recorded in both groups at the beginning of the study (T0), and after 6, 12, and at 18 months (T1, T2, T3, respectively).

Patients in the experimental group were also evaluated at the end of each 4-week MIRT.

\section{Rehabilitation treatment}

MIRT is a multidisciplinary, aerobic, motor-cognitive, intensive and goal-based rehabilitation treatment specifically designed for PD patients [13, 14, 27]. The aim of the treatment was to re-learn the dysfunctional movements resulting from the disease through the use of explicit and implicit learning strategies. It consists of a 4-week program in a hospital setting, composed of four daily rehabilitative sessions for 5 days and $1 \mathrm{~h}$ of physical exercise on the sixth day. The duration of each session, including recovery periods, is about $1 \mathrm{~h}$ :

- The first session consists of a one-to-one treatment with a physical therapist. It comprises cardiovascular warmup activities, active and passive exercises to improve the joints range of motion, stretching of the abdominal muscles, strengthening of paravertebral muscles, postural changes and exercises operating on balance and postural control.

- The second session exploits the use of various devices to improve gait and balance: a stabilometric platform with biofeedback (patients have to follow a pathway on a screen by using a cursor sensitive to their feet movements on the platform), a treadmill plus (treadmill training with visual cues, auditory cues and feedbacks) [28], a crossover [29] and a cycloergometer with feedback. We use a maximum treadmill speed of $3.5 \mathrm{Km} / \mathrm{h}$; patients are trained with treadmill for no more than $15 \mathrm{~min}$, two times per day.

- The third session consists of occupational therapy aimed to improve the autonomy in everyday activities. The session focuses on hand dexterity, writing and activities of daily living. The hand and finger dexterity training entails exercises aimed at re-acquiring the functional use of the most affected hand and the skills in the coordi- nated activities of both hands. The writing rehabilitation treatment consists of paper-and-pencil exercises and uses visual cues and verbal strategies aimed to enlarge the letters size and improve the readability. Finally, patients are trained by performing the activities of daily living in the rehabilitation setting, exploiting self-management and cognitive-behavioural strategies.

- The fourth session includes $1 \mathrm{~h}$ of speech therapy. In this field, three possible kinds of intervention are proposed: (i) a counselling for patients and caregivers pertinent to a good management of language and swallowing problems, (ii) an individual swallowing training, which includes meal monitoring and learning strategies for a correct ingestion of foods and liquids, (iii) a group therapy aimed to treat the hypokinetic dysarthria (breathing exercises to relax and alleviate the pressure of speech; facial exercises to improve the range of facial expressions and mouth motion; exercises to improve vocalization, articulation and speech prosody).

On the sixth day the patients are trained only with devices for 1 .

The rehabilitation program could also include hydrotherapy in case of severe balance and postural disorders, robotic-assisted walking training for complex gait disorders, virtual-reality training and psychoeducational groups with neuropsychologists.

During all the activities, the heart rate reserve is kept between 70 and $80 \%$.

A weekly team meeting defines the rehabilitation program for each patient and assesses its benefits during the course of the hospitalization.

\section{Statistical analysis}

The Shapiro-Wilk statistic supported by visual inspection was used to test the normality of the distribution of all variables. No severe violations of the normality assumption were observed for the considered outcome variables. Descriptive statistics of collected data were reported as mean \pm SD for continuous variables and as percentage frequency for categorical variables. Between-group comparisons for continuous variables were carried out by one-way analysis of variance. We examined differences in outcome measures using a two-factor repeated measures analysis of variance with treatment condition (Rotigotine and Rotigotine + MIRT) as the between factor and time of assessment (baseline- $-\mathrm{T} 0$, 6-months-T1, 12-months-T2 and 18 months-T3 after enrolment) as the repeated, within factor. Post-hoc analyses were performed to compare follow-up and baseline measures within each group of patients and to compare between group outcomes at T3. The Tukey-Kramer adjustment for multiple comparisons was used. Adjusted p-values were 
reported when appropriate. In the Rotigotine + MIRT group the acute effect of MIRT was evaluated at the end of the first (PostMirt1) and second 4-week treatment (PostMirt2) by repeated measures ANOVA. Gender distribution was compared between groups using the Chi square test or Fisher exact test if appropriate.

The association between changes (T3--T0) in drug dosage and in functional variables was assessed by the Spearman correlation coefficient.

A 2-sided $p$ value of 0.05 or less was considered statistically significant. All analyses were carried out using the SAS/STAT statistical package, release 9.4.

\section{Results}

Figure 1 reports the flow chart of the progress through the phases of the study. Out of 40 eligible patients approached, 4 refused to participate in the study. There were no dropouts, leading to a final population of 36 patients, 17 in Rotigotine + MIRT group and 19 in the Rotigotine group. Since all patients completed the treatment according to the original allocation, intention to treat analysis and per protocol analysis coincide. Patients' demographic characteristics are reported in Table 1. No differences in age and gender were observed across the two groups. Functional characteristics of studied patients at T0, T1, T2 and T3 observation times are reported in Table 2. The time between $\mathrm{T} 2$ and $\mathrm{T} 0$ (time distance between the two MIRT treatments in Rotigotine + MIRT group) was $12.3 \pm 1.5$ months

\section{Baseline}

Baseline values of outcome measures were not different in the two groups $(p=0.95, p=0.93, p=0.83, p=0.40$ and $p=0.73$ for total UPDRS, UPDRS III, UPDRS II, 6MWT and TUG, respectively).

Repeated measures ANOVA results for all variables are summarized in Table 3.

\section{UPDRS}

Repeated measures ANOVA revealed that the group by time interaction was not significant for all UPDRS scores (total score, III and II, $p=0.48, p=0.90$ and $p=0.40$, respectively), indicating no difference in the time course of these variables in the two groups. Indeed, total UPDRS scores in Rotigotine + MIRT group paralleled the scores in Rotigotine group and post hoc analysis revealed no significant differences at T3 in the two groups. Comparing the 95\% confidence interval for total UPDRS at T3 with the prespecified non-inferiority limit, non-inferiority was shown $(p=0.0001)$.

\section{MWT}

A significant group by time interaction was observed for 6MWT $(p<0.0001)$ indicating that the time course of this variable over 1 year and a half period was different in patients who underwent Rotigotine + MIRT compared to those treated with Rotigotine alone. Post hoc analysis showed that the value of 6MWT was higher in patients who
Table 1 Demographic characteristics of studied patients

\begin{tabular}{lllll}
\hline & All patients $(N=36)$ & $\begin{array}{l}\text { Rotigotine + MIRT } \\
(N=17)\end{array}$ & Rotigotine $(N=19)$ & $p$ value \\
\hline Age (years) & $64.5 \pm 6.0$ & $64.7 \pm 7.3$ & $64.4 \pm 4.4$ & 0.9 \\
Male gender $(\%)$ & $22(61)$ & $13(76)$ & $9(47)$ & 0.1 \\
\hline
\end{tabular}

Table 2 Clinical, motor and functional characteristics of patients at four observation times: at baseline (T0), 6 months (T1), 1 year (T2), and 18 months (T3)

\begin{tabular}{|c|c|c|c|c|c|c|c|c|}
\hline & $\begin{array}{l}\text { Rotigo- } \\
\text { tine + MIRT, } \\
\text { T0 }\end{array}$ & $\begin{array}{l}\text { Rotigo- } \\
\text { tine + MIRT, } \\
\text { T1 }\end{array}$ & $\begin{array}{l}\text { Rotigo- } \\
\text { tine + MIRT, } \\
\text { T2 }\end{array}$ & $\begin{array}{l}\text { Rotigo- } \\
\text { tine + MIRT, } \\
\text { T3 }\end{array}$ & $\begin{array}{l}\text { Rotigotine, } \\
\text { T0 }\end{array}$ & $\begin{array}{l}\text { Rotigotine, } \\
\text { T1 }\end{array}$ & $\begin{array}{l}\text { Rotigotine, } \\
\text { T2 }\end{array}$ & Rotigotine, T3 \\
\hline Total UPDRS & $30.2 \pm 8.4$ & $20.5 \pm 11.1$ & $27.8 \pm 8.0$ & $21.5 \pm 5.3$ & $30.0 \pm 7.6$ & $21.1 \pm 7.5$ & $27.7 \pm 7.4$ & $24.7 \pm 6.0$ \\
\hline UPDRS III & $18.4 \pm 5.9$ & $12.8 \pm 7.9$ & $17.2 \pm 5.1$ & $12.5 \pm 3.0$ & $18.6 \pm 4.9$ & $13.2 \pm 4.4$ & $16.9 \pm 4.8$ & $13.4 \pm 3.8$ \\
\hline UPDRS II & $8.1 \pm 3.4$ & $5.5 \pm 3.6$ & $8.5 \pm 3.8$ & $7.0 \pm 2.4$ & $8.4 \pm 3.6$ & $6.3 \pm 2.6$ & $8.8 \pm 3.1$ & $8.8 \pm 2.4$ \\
\hline $6 \mathrm{MWT}(\mathrm{m})$ & $361.2 \pm 77.3$ & $443.9 \pm 89.3$ & $414.9 \pm 87.4$ & $421.9 \pm 80.7$ & $344.7 \pm 31.2$ & $371.1 \pm 32.7$ & $372.1 \pm 30.5$ & $368.9 \pm 32.1$ \\
\hline TUG (s) & $8.8 \pm 1.7$ & $7.5 \pm 1.6$ & $8.4 \pm 2.1$ & $8.1 \pm 1.6$ & $8.5 \pm 1.2$ & $8.1 \pm 1.0$ & $8.7 \pm 0.9$ & $8.4 \pm 1.0$ \\
\hline $\begin{array}{l}\text { Rotigotine } \\
\quad(\text { dose in } \mathrm{mg})\end{array}$ & $4.0 \pm 0.0$ & $4.6 \pm 1.2$ & $5.2 \pm 1.6$ & $5.4 \pm 1.5$ & $4.0 \pm 0.0$ & $5.6 \pm 1.8$ & $6.7 \pm 1.4$ & $7.2 \pm 1.0$ \\
\hline
\end{tabular}

UPDRS Unified Parkinson's Disease Rating Scale, TUG Timed Up and Go Test, $6 M W T$ Six-Minute Walk Test, $m$ metres, $s$ seconds 
Table 3 F statistic and p-values for the main effects (Group and Time) and interaction from repeated measures ANOVA

\begin{tabular}{|c|c|c|c|c|c|c|}
\hline & \multicolumn{2}{|l|}{ Group } & \multicolumn{2}{|l|}{ Time } & \multicolumn{2}{|c|}{ Group by time } \\
\hline & $\mathrm{F}(1,34)$ & $p$ & $\mathrm{~F}(3,102)$ & $p$ & $\mathrm{~F}(3,102)$ & $p$ \\
\hline Total UPDRS & 0.16 & 0.69 & 11.01 & $<0.0001$ & 0.83 & 0.48 \\
\hline UPDRS III & 0.04 & 0.83 & 11.75 & $<0.0001$ & 0.19 & 0.90 \\
\hline UPDRS II & 0.86 & 0.36 & 6.12 & 0.0007 & 0.99 & 0.40 \\
\hline $6 \mathrm{MWT}(\mathrm{m})$ & 5.77 & 0.022 & 4.83 & 0.003 & 8.39 & $<0.0001$ \\
\hline TUG (s) & 0.66 & 0.42 & 2.54 & 0.06 & 2.95 & 0.03 \\
\hline 1-dopa eq dose & 11.19 & 0.002 & 44.11 & $<0.0001$ & 5.83 & 0.008 \\
\hline
\end{tabular}

UPDRS Unified Parkinson's Disease Rating Scale, TUG Timed Up and Go Test, 6MWT Six-Minute Walk Test, $m$ meters, $s$ seconds underwent MIRT at all observation times following T0. The difference between 6MWT values in Rotigotine + MIRT and Rotigotine at T1, T2 and T3 (unstandardized effect size) was $72.8 \mathrm{~m}(p=0.0009), 42.8 \mathrm{~m}(p=0.04)$ and $53.0 \mathrm{~m}$ $(p=0.01)$ at T1, T2 and T3, respectively.

\section{TUG}

A significant group by time interaction was observed for TUG $(p=0.03)$. Post hoc analysis revealed that the only difference in TUG in the two groups was a higher improvement between $\mathrm{T} 1$ and $\mathrm{T} 0$ in the Rotigotine + MIRT group, but the difference between TUG values at T1 was only $-0.6 \mathrm{~s}$ and did not reach statistical significance $(p=0.16)$.

\section{Rotigotine}

The time $\times$ treatment interaction was highly significant $(p=0.008)$. Post hoc analysis showed that the dosage of Rotigotine was higher in patients who did not undergo MIRT at all observation times following T0. The difference between Rotigotine dosage in Rotigotine + MIRT and Rotigotine group was $-1 \mathrm{mg}(p=0.02),-1.5 \mathrm{mg}(p=0.0002)$ and $-1.8 \mathrm{mg}(p<0.0001)$ at $\mathrm{T} 1, \mathrm{~T} 2$ and $\mathrm{T} 3$, respectively.
The total amount of Rotigotine was increased during the study in both groups, but at T3 in Rotigotine + MIRT group was increased by $30 \%$ as compared with $\mathrm{T} 0$, while in patients who did not undergo MIRT, the dosage was almost doubled, with an increase of $80 \%$.

Considering the entire study period (T3 vs T0), we found in the Rotigotine group a correlation between DRT dosage and improvements in total UPDRS and UPDRS III $(r=0.48$, $p=0.04$ and $r=0.61, p=0.005$, respectively), but none with the motor performances evaluated with $6 \mathrm{MWT}$ $(p=0.19)$ and TUG $(p=0.45)$ scores. No correlation was observed for patients in the experimental group who gained better results in motor performances $(p=0.75, p=0.83$, $p=0.55$ and $p=0.73$ for UPDRS total, UPDRS III, $6 \mathrm{MWT}$ and TUG, respectively).

We also compared the effect of the two MIRT cycles in Rotigotine + MIRT group. Results are given in Table 4. Both MIRT were effective, and the improvement was significantly better in the first cycle for all variables except TUG.

\section{Discussion}

This randomized, non-inferiority trial, aimed at comparing the short- and long-term (18 months) effectiveness of a treatment combining the use of the dopaminergic

Table 4 Changes in outcome measures in the experimental group after the first and the second MIRT

\begin{tabular}{lccccc}
\hline Variable & TPostMirt1-T0 & $p$ (TPostMirt1 vs T0) & TpostMirt2-T3 & $p$ (TpostMirt2 vs T3) & $\begin{array}{c}p \text { (TpostMirt2-T3) } \\
\text { vs (TPostMirt1-T0) }\end{array}$ \\
\hline Total UPDRS & $-14.1 \pm 5.6$ & $<0.0001$ & $-8.9 \pm 4.9$ & $<0.0001$ & 0.0009 \\
UPDRS II & $-3.9 \pm 1.9$ & $<0.0001$ & $-2.6 \pm 1.8$ & $<0.0001$ & 0.040 \\
UPDRS III & $-8.7 \pm 4.3$ & $<0.0001$ & $-6.1 \pm 5.1$ & 0.0002 & 0.020 \\
TUG (s) & $-1.7 \pm 1.0$ & $<0.0001$ & $-1.1 \pm 0.9$ & $<0.0001$ & 0.14 \\
6MWT (m) & $81.8 \pm 50.7$ & $<0.0001$ & $48.1 \pm 29.9$ & $<0.0001$ & 0.038 \\
\hline
\end{tabular}

TpostMIRT1vsT0: improvement after the first rehabilitation treatment; TpostMIRT2vsT3: improvement after the second rehabilitation treatment; TpostMIRT1-T3 vs TpostMIRT1-T1: differences between the improvement after the first and the second rehabilitation treatment

UPDRS Unified Parkinson's Disease Rating Scale, TUG Timed Up and Go Test, 6MWT Six-Minute Walk Test, $m$ meters, $s$ seconds, TpostMIRT Time Post Multidisciplinary Intensive Rehabilitation Treatment 
drug Rotigotine plus rehabilitation versus a pharmacological treatment alone with the same DA, in "de-novo" PD patients who have never been previously treated with any other dopaminergic drug.

\section{Main findings and novelties for the field}

Five main findings raise from this study: (i) the effectiveness of the combined treatment (Rotigotine plus MIRT) on the patients' global clinical status, evaluated with total UPDRS, was not inferior to that of the pharmacological treatment alone with Rotigotine; (ii) patients in the Rotigotine + MIRT group gained better performances at 6MWT and TUG; (iii) improvements in clinical performances were achieved with lower DA dose in comparison to the control group, so that patients who did undergo rehabilitation needed to increase dopaminergic drug dosage by $50 \%$ less than those treated only with the pharmacological therapy; (iv) considering the entire study period (T3 vs T0), we found in the Rotigotine group a direct correlation between DRT dosage and improvements in total UPDRS and UPDRS III, but we did not find a direct correlation with the motor performances evaluated with 6MWT and TUG scores. No correlation was observed for patients in the experimental group who gained better results in motor performances; (v) the improvement we found in gait performances (evaluated with 6MWT) was higher than the MDC (equal to $82 \mathrm{~m}$, according to Steffen and Seney [30]), in 52.9\% of subjects in the experimental group, while no one of those in the control group achieved this result, during the first study period.

As different preclinical or subclinical motor abnormalities have been found in early PD [31-33], these data appear to be even more relevant.

These results confirm the beneficial role of exercise for "de-novo" PD subjects: Christiansen et al. [34] have recently proposed that clinicians should consider the costs and benefits of exercise and activity behaviour interventions immediately after the diagnosis of PD. For these authors, this is essential to attenuate the health consequences of habitual low walking activity, that is typical in parkinsonian subjects compared with healthy people of similar age $[35,36]$. Specifically, they found that step count in people with "de-novo" PD who were not regular exercisers approached sedentary lifestyle levels [34], thus suggesting that improving cardiorespiratory fitness might be an effective strategy to maintain healthy levels of walking activity and improve cardiorespiratory fitness [34]. In 16-month randomized controlled exercise intervention, Schenkman et al. [37] investigated three exercise approaches in individuals with early or mild stage PD: flexibility/balance/function exercise, supervised aerobic exercise, and home-based exercise. They showed overall functional benefits at 4 months in the flexibility/ balance/function exercise group and improved walking economy (up to 16 months) in the aerobic exercise group [37]. It has been also observed that people with early PD participating in high-intensity body weight- supported treadmill training improve spatiotemporal gait parameters, kinematics of gait performance, and lower-extremity symmetry of ground reaction force in sit-to-stand task [23]. Consistently, it has been recently proposed that high-intensity treadmill exercise may be feasible and prescribed safely for patients with "de-novo" PD, but the authors conclude that an efficacy trial is warranted to determine whether high-intensity treadmill exercise produces meaningful clinical benefits in these patients $[38,39]$. Beyond the different design and results, these studies were limited by the lack of a multidisciplinary, integrated motor-cognitive approach, which has been proposed to our patients and that is considered as central in the clinical management of PD and for patients' quality of life [40-42]. It is also relevant that for this study we focused on individuals with "de-novo" PD and naïve to therapy, as it allows not only minimizing the confounding effects of medication on exercise intervention (as well as the confounding effect of other drugs different from Rotigotine for subjects in the Rotigotine group) but also the likelihood that patients would have functional limitations precluding exercise [39].

\section{Effectiveness of MIRT and Rotigotine: proposed mechanisms}

Rehabilitation could be considered complementary to DRT and effective for the management of PD [10-16], especially for its positive effect on postural, balance and gait dysfunctions, well-known for their DRT-resistance [17-19]. Nevertheless, its essential role also for early PD subjects has already been widely described [14]. DRT partially improves the PD disturbances, mainly bradykinesia, tremor and rigidity [9]: these aspects represent only a part of the wide whole of disturbances that compose the PD spectrum [9], as the disease results in cognitive-motor abnormalities and in disorders of both movement expression and action performing. The main feature of the disease is the impaired ability to learn and express habitual-automatic actions [43-45]: since DRT does not improve the expression of habitual-automatic actions in Parkinsonian subjects, the main goal of rehabilitation should be properly the re-learning of the lost habitual motor behaviours. In fact, even though their habitual scheduling is altered [43], the parkinsonian subjects can still express habitual skills by using the executive-volitional component of action [28, 46-49]. For this reason, in the field of PD rehabilitation, external stimuli and specific techniques and strategies [28, 34-36, 50] have been properly developed in order to perform motor actions in a volitional and goaldirected manner [13, 14, 28, 49]. 
The improvements in motor performances following rehabilitation in PD could be related to activity-dependent neuroplastic changes [22, 51, 52]. Different variables, such as intensity, specificity, difficulty and complexity of exercise, have been recognized as fundamental for evoking this "activity-dependent neuroplasticity" in neurodegenerative disorders, including PD [53-55]. Nevertheless, the improvements in motor performances after MIRT cannot be explained only by these "activity-dependent" processes. The goal-directed approach of this kind of multidisciplinary and intensive rehabilitation is another fundamental requirement for achieve motor-functional improvements [49]. The physical techniques, the devices and the cognitive strategies adopted in this rehabilitative protocol, exploit the functions of the frontal cortical regions (specifically the pre-frontal cortex) and allow: (i) to bypass the dysfunctional basal ganglia, (ii) to stimulate the re-learning of the lost automatic movements, (iii) to reinforce the cortical mechanisms involved in the execution of the commands to move and, finally, (iv) to improve the patients' mobility, balance, gait and posture [46, 49, 50, 56, 57].

Not least, it is conceivable that making exercise promotes proactive and resilient attitudes that could increase the patients" sense of "self-management" of the disease.

With regard to Rotigotine, this DA not only offers advantages in term of onset of action, drug delivery and ease of use $[7,8]$, but it seems to have also a neuroprotective effect, as it has been demonstrated in the acute MPTP (1-methyl4-phenyl-1,2,3,6-tetrahydropyridine) lesioned mouse model of PD [58]. Therefore, it is arguable that the use of Rotigotine possibly might maximize the benefits of motor exercises in terms of neuroprotection and neuroplasticity.

\section{The role of the synergism between DRT use and rehabilitative efforts}

These results could be interpreted as a clear example of a good synergism between DRT use and rehabilitative efforts: the total amount of DRT at the end of the study was lower in patients who underwent rehabilitation, thus suggesting that the benefits obtained with MIRT reduce the need for increasing the dopaminergic drug dosage. Based on our results we hypothesize that MIRT plus DRT may delay or eliminate DRT side effects. Moreover, an optimal DRT titration is by itself fundamental for obtaining gains from rehabilitation: indeed, despite both DRT and different activity-dependent processes could restore the physiological synaptic plasticity in the dopamine-denervated striatum $[1,20,54]$, the incautious and wrong DRT use could negatively influence the learning and reward processes, which are essential for rehabilitative purpose [59].

\section{Study limitations}

There are some limitations of this study that have to be acknowledged. First of all, the design of this study could be considered as a limitation, due to the possible placebo effect. The placebo effect in PD is mediated by the release of dopamine in the dorsal striatum: the expectations of reward, such as those related to a novel pharmacological treatment or rehabilitation, seem to be particularly relevant for the "placebo-induced" clinical benefits [60, 61]. These benefits are strictly related to dopamine release in the ventral striatum, which leads to the activation of the reward circuitry [62]. The magnitude of the placebo-induced response likely depends on the "a priori" probability of clinical benefits [62]. This notion has profound implication in the design of clinical trials for PD [62]. Nevertheless, MIRT has been tested in previous studies and its effectiveness on motor, clinical and functional outcomes has been found to be closely related with objective metabolic measures [63] and with changes in the plasmatic levels of biochemical and molecular markers of neuroplasticity [22] so that the hypothesis of a placebo effect on outcome measures is unlikely.

Further, the study size is small, and this represents another limitation of the present study.

We did not use specific outcome measures for speech and dexterity despite the evidence that the intervention is a result of the total package, with presumed outcomes well beyond mobility. This should be considered as a study limitation. Nevertheless, among the secondary outcomes, we chose the total UPDRS, which allows evaluating the motor performance and different activities of daily life such as eating, dressing, writing, talking and the dexterity. Certainly, future studies with more appropriate outcome measures that more directly evaluate the effect of occupational therapy or speech therapy should be designed.

Finally, we did not perform any non-specific, non-intensive conventional rehabilitation in another group of PD patients in order to evaluate whether our results were related to the specific features of MIRT or could be interpreted as a general effects of exercise. Nevertheless, a number of literature data confirm how specific exercise features in terms of intensity and specificity are important to induce activityrelated neuroplastic changes and clinical benefits [20-23, $51,52,54]$.

\section{Conclusion}

We observed in a group of "de-novo" Parkinsonian subjects that, over the course of 18 months, the effectiveness of a combined treatment, Rotigotine plus MIRT, on the patients' global clinical status was not inferior to that of 
the pharmacological treatment with Rotigotine alone. Nevertheless, over the time course, patients who underwent rehabilitation gained better performances at 6MWT and TUG and, above all, their need for increasing DRT was lower in comparison with patients treated only with the pharmacological therapy. These results underpin the importance of the synergism between DRT and rehabilitation in reducing the impact of the disease and the severity of symptoms, in controlling the short- and long-term DRT side effects and, finally, in promoting a pro-active attitude for the self management of the disease.

Authors' contributions DF: data acquisition; conception, design; drafting the article; revising the article critically, PO: data acquisition; conception, design; drafting the article; revising the article critically, GR: data acquisition; conception, design; final approval of the version to be submitted, RM: analysis, interpretation; revising the article critically; final approval of the version to be submitted, GF: conception, design; data acquisition; analysis, interpretation; drafting the article; revising the article critically; final approval of the version to be submitted

Funding This research did not receive any specific grant from funding agencies in the public, commercial, or not-for-profit sectors.

\section{Compliance with ethical standards}

Conflicts of interest All authors declare that there is no conflict of interest regarding this work. All authors have approved the final article.

Ethical standard The study design and protocol were approved by the by the local institutional review board and by the Central Ethics Committee and were in accordance with the code of Ethics of the World Medical Association (Declaration of Helsinki, 1967).

Informed consent All patients signed an informed written consent prior to the participation to the study for the use of their clinical data for scientific purposes.

Open Access This article is distributed under the terms of the Creative Commons Attribution 4.0 International License (http://creativeco mmons.org/licenses/by/4.0/), which permits unrestricted use, distribution, and reproduction in any medium, provided you give appropriate credit to the original author(s) and the source, provide a link to the Creative Commons license, and indicate if changes were made.

\section{References}

1. Calabresi P, Ghiglieri V, Mazzocchetti P, Corbelli I, Picconi B (2015) Levodopa-induced plasticity: a double-edged sword in Parkinson's disease? Philos Trans R Soc Lond B Biol Sci 370(1672). https://doi.org/10.1098/rstb.2014.0184 (pii: 20140184)

2. Jankovic J (2008) Parkinson's disease: clinical features and diagnosis. J Neurol Neurosurg Psychiatry 79:368-376. https://doi. org/10.1136/jnnp.2007.131045

3. Nishijima H, Suzuki S, Kon T, Funamizu Y, Ueno T, Haga R, Suzuki C, Arai A, Kimura T, Suzuki C, Meguro R, Miki Y, Yamada J, Migita K, Ichinohe N, Ueno S, Baba M, Tomiyama
M (2014) Morphologic changes of dendritic spines of striatal neurons in the levodopa-induced dyskinesia model. Mov Disord 29:336-343. https://doi.org/10.1002/mds.25826

4. Chapuis S, Ouchchane L, Metz O, Gerbaud L, Durif F (2005) Impact of the motor complications of Parkinson's disease on the quality of life. Mov Disord 20:224-230

5. Warren Olanow C, Kieburtz K, Rascol O, Poewe W, Schapira AH, Emre M, Nissinen H, Leinonen M, Stocchi F (2013) Factors predictive of the development of Levodopa-induced dyskinesia and wearing-off in Parkinson's disease. Mov Disord 28:1064-1071. https://doi.org/10.1002/mds.25364

6. Stowe RL, Ives NJ, Clarke C, van Hilten J, Ferreira J, Hawker RJ, Shah L, Wheatley K, Gray R (2008) Dopamine agonist therapy in early Parkinson's disease. Cochrane Database Syst Rev 2:CD006564. https://doi.org/10.1002/14651858.cd006564. pub2

7. Poewe WH, Rascol O, Quinn N, Tolosa E, Oertel WH, Martignoni E, Rupp M, Boroojerdi B, SP 515 Investigators (2007) Efficacy of pramipexole and transdermal rotigotine in advanced Parkinson's disease: a double-blind, double-dummy, randomised controlled trial. Lancet Neurol 6:513-520

8. Antonini A, Bernardi L, Calandrella D, Mancini F, Plebani M (2010) Rotigotine transdermal patch in the management of Parkinson's disease (PD) and its night-time use for PD-related sleep disorders. Funct Neurol 25:21-25

9. Lang AE, Obeso JA (2004) Challenges in Parkinson's disease: restoration of the nigrostriatal dopamine system is not enough. Lancet Neurol 3:309-316

10. Tomlinson CL, Patel S, Meek C, Herd CP, Clarke CE, Stowe R, Shah L, Sackley CM, Deane KH, Wheatley K, Ives N (2013) Physiotherapy versus placebo or no intervention in Parkinson's disease. Cochrane Database Syst Rev 9:CD002817. https://doi. org/10.1002/14651858.cd002817.pub4

11. Bloem BR, de Vries NM, Ebersbach G (2015) Nonpharmacological treatments for patients with Parkinson's disease. Mov Disord 30:1504-1520. https://doi.org/10.1002/mds.26363

12. Goodwin VA, Richards SH, Taylor RS, Taylor AH, Campbell JL (2008) The effectiveness of exercise interventions for people with Parkinson's disease: a systematic review and meta-analysis. Mov Disord 23:631-640. https://doi.org/10.1002/mds.21922

13. Frazzitta G, Bertotti G, Riboldazzi G, Turla M, Uccellini D, Boveri N, Guaglio G, Perini M, Comi C, Balbi P, Maestri R (2012) Effectiveness of intensive inpatient rehabilitation treatment on disease progression in parkinsonian patients: a randomized controlled trial with 1-year follow-up. Neurorehabil Neural Repair 26:144-150. https://doi.org/10.1177/1545968311416990

14. Frazzitta G, Maestri R, Bertotti G, Riboldazzi G, Boveri N, Perini M, Uccellini D, Turla M, Comi C, Pezzoli G, Ghilardi MF (2015) Intensive rehabilitation treatment in early Parkinson's disease: a randomized pilot study with a 2-year follow-up. Neurorehabil Neural Repair 29:123-131. https://doi.org/10.1177/1545968314 542981

15. Ekker MS, Janssen S, Nonnekes J, Bloem BR, de Vries N (2016) Neurorehabilitation for Parkinson's disease: future perspectives for behavioural adaptation. Parkinsonism Relat Disord 22(Suppl 1):73-77. https://doi.org/10.1016/j.parkreldis.2015.08.031

16. Keus SH, Munneke M, Nijkrake MJ, Kwakkel G, Bloem BR (2009) Physical therapy in Parkinson's disease: evolution and future challenges. Mov Disord 24:1-14. https://doi.org/10.1002/ mds. 22141

17. Canning CG, Sherrington C, Lord SR, Close JC, Heritier S, Heller GZ, Howard K, Allen NE, Latt MD, Murray SM, O'Rourke SD, Paul SS, Song J, Fung VS (2015) Exercise for falls prevention in Parkinson disease: a randomized controlled trial. Neurology 84:304-312. https://doi.org/10.1212/WNL.0000000000001155 
18. Smania N, Corato E, Tinazzi M, Stanzani C, Fiaschi A, Girardi P, Gandolfi M (2010) Effect of balance training on postural instability in patients with idiopathic Parkinson's disease. Neurorehabil Neural Repair 24:826-834. https://doi.org/10.1177/1545968310 376057

19. Nieuwboer A, Kwakkel G, Rochester L, Jones D, van Wegen E, Willems AM, Chavret F, Hetherington V, Baker K, Lim I (2007) Cueing training in the home improves gait-related mobility in Parkinson's disease: the RESCUE trial. J Neurol Neurosurg Psychiatry $78: 134-140$

20. Petzinger GM, Fisher BE, Van Leeuwen JE, Vukovic M, Akopian G, Meshul CK, Holschneider DP, Nacca A, Walsh JP, Jakowec MW (2010) Enhancing neuroplasticity in the basal ganglia: the role of exercise in Parkinson's disease. Mov Disord 25(Suppl 1):S141-S145. https://doi.org/10.1002/mds.22782

21. Petzinger GM, Fisher BE, McEwen S, Beeler JA, Walsh JP, Jakowec MW (2013) Exercise-enhanced neuroplasticity targeting motor and cognitive circuitry in Parkinson's disease. Lancet Neurol 12:716-726. https://doi.org/10.1016/S1474 -4422(13)70123-6

22. Fontanesi C, Kvint S, Frazzitta G, Bera R, Ferrazzoli D, Di Rocco A, Rebholz H, Friedman E, Pezzoli G, Quartarone A, Wang HY, Ghilardi MF (2016) Intensive rehabilitation enhances lymphocyte BDNF-TrkB signaling in patients with Parkinson's disease. Neurorehabil Neural Repair 30:411-418. https://doi. org/10.1177/1545968315600272

23. Fisher BE, $\mathrm{Wu} \mathrm{AD}$, Salem GJ, Song J, Lin CH, Yip J, Cen S, Gordon J, Jakowec M, Petzinger G (2008) The effect of exercise training in improving motor performance and corticomotor excitability in people with early Parkinson's disease. Arch Phys Med Rehabil 89:1221-1229. https://doi.org/10.1016/j.apmr.2008.01.013

24. Ferrazzoli D, Carter A, Ustun FS, Palamara G, Ortelli P, Maestri R, Yücel M, Frazzitta G (2016) Dopamine replacement therapy, learning and reward prediction in Parkinson's disease: implications for rehabilitation. Front Behav Neurosci 10:121. https://doi. org/10.3389/fnbeh.2016.00121

25. Hughes AJ, Daniel SE, Kilford L, Lees AJ (1992) Accuracy of clinical diagnosis of idiopathic Parkinson's disease: a clinicopathological study of 100 cases. J Neurol Neurosurg Psychiatry 55:181-184

26. Shulman LM, Gruber-Baldini AL, Anderson KE, Fishman PS, Reich SG, Weiner WJ (2010) The clinically important difference on the unified Parkinson's disease rating scale. Arch Neurol 67:64-70. https://doi.org/10.1001/archneurol.2009.295

27. Frazzitta G, Bertotti G, Uccellini D, Maestri R (2010) Parkinson's disease rehabilitation: a pilot study with 1 year follow up. Mov Disord 25:1762-1763. https://doi.org/10.1002/mds.23316

28. Frazzitta G, Maestri R, Uccellini D, Bertotti G, Abelli P (2009) Rehabilitation treatment of gait in patients with Parkinson's disease with freezing: a comparison between two physical therapy protocols using visual and auditory cues with or without treadmill training. Mov Disord 24:1139-1143. https://doi.org/10.1002/ mds.22491

29. Frazzitta G, Bossio F, Maestri R, Palamara G, Bera R, Ferrazzoli D (2015) Crossover versus stabilometric platform for the treatment of balance dysfunction in Parkinson's disease: a randomized study. Biomed Res Int 2015:878472. https://doi.org/10.1155/2015/87847 2

30. Steffen T, Seney M (2008) Test-retest reliability and minimal detectable change on balance and ambulation tests, the 36-item short-form health survey, and the unified Parkinson disease rating scale in people with parkinsonism. Phys Ther 88:733-746. https ://doi.org/10.2522/ptj.20070214

31. Panyakaew P, Bhidayasiri R (2013) The spectrum of preclinical gait disorders in early Parkinson's disease: subclinical gait abnormalities and compensatory mechanisms revealed with dual tasking. J Neural Trans 120:1665-1672. https://doi.org/10.1007/ s00702-013-1051-8

32. Ganesan M, Pal PK, Gupta A, Sathyaprabha TN (2010) Dynamic posturography in evaluation of balance in patients of Parkinson's disease with normal pull test: concept of a diagonal pull test. Parkinsonism Relat Disord 16:595-599. https://doi.org/10.1016/j. parkreldis.2010.08.005

33. Ferrazzoli D, Fasano A, Maestri R, Bera R, Palamara G, Ghilardi MF, Pezzoli G, Frazzitta G (2015) Balance dysfunction in Parkinson's disease: the role of posturography in developing a rehabilitation program. Parkinsons Dis 2015:520128. https:// doi.org/10.1155/2015/520128

34. Christiansen C, Moore C, Schenkman M, Kluger B, Kohrt W, Delitto A, Berman B, Hall D, Josbeno D, Poon C, Robichaud J, Wellington T, Jain S, Comella C, Corcos D, Melanson E (2017) Factors associated with ambulatory activity in de novo Parkinson disease. J Neurol Phys Ther 41:93-100. https://doi. org/10.1097/NPT.0000000000000169

35. van Nimwegen M, Speelman AD, Hofman-van Rossum EJ, Overeem S, Deeg DJ, Borm GF, van der Horst MH, Bloem BR, Munneke M (2011) Physical inactivity in Parkinson's disease. J Neurol 258:2214-2221. https://doi.org/10.1007/s0041 5-011-6097-7

36. Lord S, Godfrey A, Galna B, Mhiripiri D, Burn D, Rochester L (2013) Ambulatory activity in incident Parkinson's: more than meets the eye? J Neurol 260:2672-2964. https://doi. org/10.1007/s00415-013-7037-5

37. Schenkman M, Hall DA, Barón AE, Schwartz RS, Mettler P, Kohrt WM (2012) Exercise for people in early- or mid-stage Parkinson disease: a 16-month randomized controlled trial. Phys Ther 92:1395-1410. https://doi.org/10.2522/ptj.20110472

38. Schenkman M, Moore CG, Kohrt WM, Hall DA, Delitto A, Comella CL, Josbeno DA, Christiansen CL, Berman BD, Kluger BM, Melanson EL, Jain S, Robichaud JA, Poon C, Corcos DM (2017) Effect of high-intensity treadmill exercise on motor symptoms in patients with de novo Parkinson disease: a phase 2 randomized clinical trial. JAMA Neurol. https://doi. org/10.1001/jamaneurol.2017.3517 (Epub ahead of print)

39. Moore CG, Schenkman M, Kohrt WM, Delitto A, Hall DA, Cor$\cos$ D (2013) Study in Parkinson disease of exercise (SPARX): translating high-intensity exercise from animals to humans. Contemp Clin Trial 36:90-98. https://doi.org/10.1016/j. cct.2013.06.002

40. Giladi N, Manor Y, Hilel A, Gurevich T (2014) Interdisciplinary teamwork for the treatment of people with Parkinson's disease and their families. Curr Neurol Neurosci Rep 14:493. https:// doi.org/10.1007/s11910-014-0493-1

41. Rochester L, Espay AJ (2015) Multidisciplinary rehabilitation in Parkinson's disease: a milestone with future challenges. Mov Disord 30:1011-1013. https://doi.org/10.1002/mds.26277

42. Ferrazzoli D, Ortelli P, Zivi I, Cian V, Urso E, Ghilardi MF, Maestri R, Frazzitta G (2018) Efficacy of intensive multidisciplinary rehabilitation in Parkinson's disease: a randomised controlled study. J Neurol Neurosurg Psychiatry. https://doi. org/10.1136/jnnp-2017-316437 (pii: jnnp-2017-316437)

43. Beeler JA, Petzinger G, Jakowec MW (2013) The Enemy within: propagation of aberrant corticostriatal learning to cortical function in Parkinson's disease. Front Neurol 4:134. https://doi. org/10.3389/fneur.2013.00134

44. Redgrave P, Rodriguez M, Smith Y, Rodriguez-Oroz MC, Lehericy S, Bergman H, Agid Y, DeLong MR, Obeso JA (2010) Goal-directed and habitual control in the basal ganglia: implications for Parkinson's disease. Nat Rev Neurosci 11:760-772. https://doi.org/10.1038/nrn2915

45. Jahanshahi M, Obeso I, Rothwell JC, Obeso JA (2015) A fronto-striato-subthalamic-pallidal network for goal-directed 
and habitual inhibition. Nat Rev Neurosci 16:719-732. https:// doi.org/10.1038/nrn4038

46. Morris ME, Iansek R, Kirkwood B (2009) A randomized controlled trial of movement strategies compared with exercise for people with Parkinson's disease. Mov Disord 24:64-71. https ://doi.org/10.1002/mds.22295

47. Morris ME, Iansek R, Matyas TA, Summers JJ (1994) The pathogenesis of gait hypokinesia in Parkinson's disease. Brain 117:1169-1181

48. Morris ME, Iansek R, Matyas TA, Summers JJ (1996) Stride length regulation in Parkinson's disease. Normalization strategies and underlying mechanisms. Brain 119:551-568

49. Ferrazzoli D, Ortelli P, Maestri R, Bera R, Gargantini R, Palamara G, Zarucchi M, Giladi N, Frazzitta G (2017) Focused and sustained attention is modified by a goal-based rehabilitation in Parkinsonian patients. Front Behav Neurosci 11:56. https://doi. org/10.3389/fnbeh.2017.00056

50. Morris ME, Martin CL, Schenkman ML (2010) Striding out with Parkinson disease: evidence-based physical therapy for gait disorders. Phys Ther 90:280-288. https://doi.org/10.2522/ptj.20090 091

51. Hirsch MA, Iyer SS, Sanjak M (2016) Exercise-induced neuroplasticity in human Parkinson's disease: what is the evidence telling us? Parkinsonism Relat Disord 22(Suppl 1):S78-S81. https:// doi.org/10.1016/j.parkreldis.2015.09.030

52. Fisher BE, Li Q, Nacca A, Salem GJ, Song J, Yip J, Hui JS, Jakowec MW, Petzinger GM (2013) Treadmill exercise elevates striatal dopamine D2 receptor binding potential in patients with early Parkinson's disease. NeuroReport 24:509-514. https://doi. org/10.1097/WNR.0b013e328361dc13

53. Fisher BE, Petzinger GM, Nixon K, Hogg E, Bremmer S, Meshul CK, Jakowec MW (2004) Exercise-induced behavioral recovery and neuroplasticity in the 1-methyl-4-phenyl-1,2,3,6tetrahydropyridine-lesioned mouse basal ganglia. J Neurosci Res 77:378-390

54. Petzinger GM, Walsh JP, Akopian G, Hogg E, Abernathy A, Arevalo P, Turnquist P, Vucković M, Fisher BE, Togasaki DM,
Jakowec MW (2007) Effects of treadmill exercise on dopaminergic transmission in the 1-methyl-4-phenyl-1,2,3,6-tetrahydropyridine-lesioned mouse model of basal ganglia injury. J Neurosci 27:5291-5300

55. Tillerson JL, Cohen AD, Philhower J, Miller GW, Zigmond MJ, Schallert T (2001) Forced limb-use effects on the behavioral and neurochemical effects of 6-hydroxydopamine. J Neurosci 21:4427-4435

56. Morris ME, Iansek R, Galna B (2008) Gait festination and freezing in Parkinson's disease: pathogenesis and rehabilitation. Mov Disord 23(Suppl 2):S451-S460. https://doi.org/10.1002/ mds. 21974

57. Morris ME (2006) Locomotor training in people with Parkinson disease. Phys Ther 86:1426-1435

58. Scheller D, Stichel-Gunkel C, Lübbert H, Porras G, Ravenscroft P, Hill M, Bezard E (2008) Neuroprotective effects of rotigotine in the acute MPTP-lesioned mouse model of Parkinson's disease. Neurosci Lett 432:30-34

59. Ferrazzoli D, Carter A, Ustun FS, Palamara G, Ortelli P, Maestri R, Yücel M, Frazzitta G (2016) Dopamine replacement therapy, learning and reward prediction in Parkinson's Disease: implications for rehabilitation. Front Behav Neurosci 10:121. https://doi. org/10.3389/fnbeh.2016.00121

60. de la Fuente-Fernández R, Stoessl AJ (2002) The placebo effect in Parkinson's disease. Trends Neurosci 25:302-306

61. de la Fuente-Fernández R (2009) The placebo-reward hypothesis: dopamine and the placebo effect. Parkinsonism Relat Disord 15(Suppl 3):S72-S74. https://doi.org/10.1016/S1353 $-8020(09) 70785-0$

62. de la Fuente-Fernández R, Schulzer M, Stoessl AJ (2004) Placebo mechanisms and reward circuitry: clues from Parkinson's disease. Biol Psychiatry 56:67-71

63. Frazzitta G, Maestri R, Bertotti G, Uccellini D, Bazzini G, Abelli $P$, Aquilani R (2010) Rehabilitation in Parkinson's disease: assessing the outcome using objective metabolic measurements. Mov Disord 25:609-614. https://doi.org/10.1002/mds.22871 